\title{
Correlation between Acoustic Parameters and Voice Handicap Index in Dysphonic Teachers
}

\author{
E. Niebudek-Bogusz E. Woznicka E. Zamyslowska-Szmytke \\ M. Sliwinska-Kowalska \\ Department of Audiology and Phoniatrics, Nofer Institute of Occupational Medicine, Lodz, Poland
}

\author{
Key Words \\ Occupational dysphonia • Voice Handicap Index • \\ Biopsychosocial implications of voice problems · Acoustic \\ voice analysis
}

\begin{abstract}
Objective: The aim of this study was to investigate the relationship between acoustic analysis and biopsychosocial implications of voice problems, evaluated by the Voice Handicap Index (VHI). Materials and Methods: The study comprised 120 female teachers with voice disorders, evaluated by videolaryngostroboscopy. $60.8 \%$ of this group were diagnosed as having functional dysphonia and $39.2 \%$ had dysphonia with benign vocal fold masses (nodules and polyps). The controls consisted of 30 euphonic women. The correlations between VHI and acoustic analysis were assessed in both groups using the Pearson correlation coefficient and regression analysis. Results: In teachers, the total VHI score was over 5 times as high as in controls ( $p<0.001)$. Moreover, in teachers, significant positive correlations were found between the total VHI score and the frequency perturbation parameters and amplitude perturbation parameters when both statistical methods were used. These acoustic parameters also significantly correlated with the score on the func-
\end{abstract}

tional and emotional subscales, but rarely with the physical subscale of the VHI. Conclusions: The study revealed a significant relationship between the objective voice measurements and the VHI. The results confirmed that VHI can be a valuable tool for assessing biopsychosocial implications of occupational dysphonia and should be incorporated in multidimensional voice evaluation.

Copyright $\odot 2010$ S. Karger AG, Base

\section{Introduction}

Currently, significant efforts have been made to develop objective measures of voice quality in an attempt to quantify the severity of voice pathology [1-3]. However, neither the objective evaluation via acoustic or aerodynamic measurements nor the technologically advanced images of the larynx (videolaryngostroboscopy, kymography, or high-speed videoendoscopy) make it possible to assess the level of handicap that the patient experiences as a result of voice disorders $[4,5]$. In reference to the WHO multidimensional concept of health as physical, mental and social well-being, it has been important to measure how much one's quality of life has deteriorated due to dysphonia defined as an array of self-reported

\section{KARGER \\ Fax +4161306 1234 \\ E-Mail karger@karger.ch}

www.karger.com
(C) 2010 S. Karger AG, Basel

$1021-7762 / 10 / 0622-0055 \$ 26.00 / 0$

Accessible online at:

www.karger.com/fpl
Ewa Niebudek-Bogusz, MD, $\mathrm{PhD}$

Department of Audiology and Phoniatrics

Nofer Institute of Occupational Medicine, ul. Teresy 8

PL-91-348 Lodz (Poland)

Tel. +48 42631 4545, Fax +48 42631 4519, E-Mail ebogusz@imp.lodz.pl 


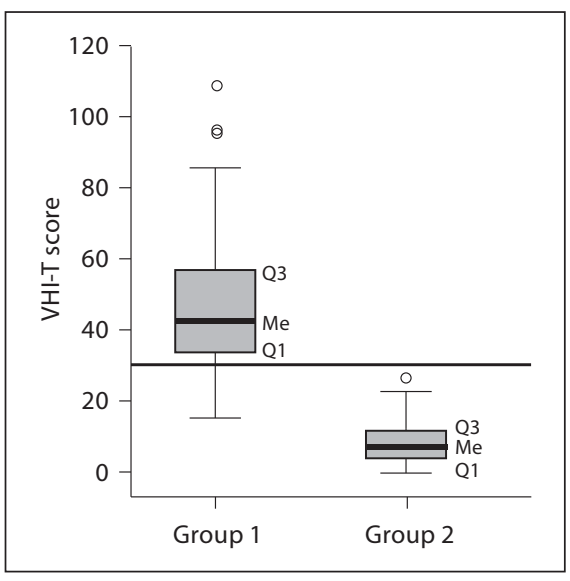

Fig. 1. The boxplots of the VHI-T score in teachers (group 1) and controls (group 2). $\mathrm{Me}=$ Median; Q1 = 25th percentile; Q3 = 75th percentile. The reference value at VHI $=30$ is indicated.

symptoms and clinically observed signs of pathological voice production [6]. This definition includes a large degree of subjectivity in the identification of voice disorders $[7,8]$. Therefore, if the patient reports functional disruption in voice, his/her opinion should not be ignored in assessing the health consequence of voice disorders. The measurement of voice handicap ought to be taken into account particularly among professional voice users (e.g. teachers), for whom voice disorders may not only have an impact on their general health condition but also bring about employment-related problems [9-11]. One of the most common instruments of self-assessment that has been designed to evaluate the quality of life specific to dysphonia is the Voice Handicap Index (VHI). This scale has been shown to have construct validity and reliability [12]. Recent international projects have demonstrated that the VHI results obtained from studies conducted in different countries are comparable [13].

The purpose of the present study was to examine the correlation between the VHI and objective voice measurements, as well as comparing the results of the VHI and of the acoustic analysis obtained in dysphonic teachers with the controls.

\section{Methods}

The study covered 120 female teachers (mean age 44.4 years, mean teaching experience 19.3 years) with voice disorders. The controls were 30 women with euphonic voice (mean age 40.6 years), whose job did not involve vocal effort.
All the participants filled in the VHI, and were subjected to laryngological examination and acoustic voice analysis. The VHI consists of 30 statements, scored from 0 to 4 , that measure the physical, emotional and functional aspects of voice on respective subscales (VHI-P, VHI-E, VHI-F). A low total VHI score (VHI-T: score $0-30$ ) relates to light voice problems, a score of 30-60 represents a medium-to-heavy handicap level, and a score ranging from 61 to 120 indicates severe voice disorders and an urgent necessity for additional voice assessments.

In teachers, clinical examinations comprised routine laryngological examination followed by videolaryngostroboscopy. Voice assessment in both groups involved perceptual evaluation and determination of the aerodynamic parameter: maximum phonation time (MPT). The following acoustic parameters of sustained vowel phonation were assessed: (1) frequency perturbation parameters: pitch perturbation (jitter), relative average perturbation (RAP) and pitch perturbation quotient (PPQ); (2) amplitude perturbation parameters: amplitude perturbation (shimmer) and amplitude perturbation quotient (APQ), and (3) noise evaluation measurement: noise-to-harmonic ratio (NHR). The examination procedure has been described in detail elsewhere [14].

Statistical analysis was performed using the SPSS software (version 14.0). The correlations between the VHI-T score or the score on any of the VHI subscales and the objective voice parameters were calculated with Pearson's coefficient and regression analysis, and $p<0.05$ was considered the level of significance. The distribution of the variables was examined and no major deviations from the norm were observed.

\section{Results}

In teachers, videolaryngostroboscopy revealed various voice pathologies. Most frequently (60.8\%) diagnosed was functional dysphonia (prenodules, glottal insufficiency with bowed vocal folds, hyperfunctional dysphonia with vestibular voice). Less frequently recognized (39.2\%) were benign vocal fold masses (BVFM), such as vocal nodules (24.2\%) and small polyps of vocal fold margin (15\%). The analysis of the VHI score revealed that $62 \%$ of the teachers (group 1) assessed their own voice problems as a medium-to-heavy handicap, while $20 \%$ of the teachers reported severe voice handicap. Only $18 \%$ of the teachers evaluated their voice problems as light, compared with the control group (group 2) in which $100 \%$ of the subjects had a low VHI score. Among the teachers who assessed their voice problems as severe, $75 \%$ were those with BVFM. A more detailed analysis of the VHI-T scores in both groups (fig. 1) confirmed the significant differences between the teachers and controls. The values found for controls are much below the upper limit of the standard value (VHI-T score $=30$ ), marked as a horizontal line in the diagram, while those noted for the teachers are significantly higher (in some cases even 5 times as high as the standard). Moreover, with regard to the find- 
ings on the subscales of the VHI (VHI-F, VHI-E, VHI-P), teachers had a score over 5-7 times higher $(p<0.001)$ on the particular subscales than controls (fig. 2). The most distinct differences were noted on the physical subscale. The MPT and acoustic parameters also differed significantly in both groups (table 1).

The analysis of the relationship between the MPT parameter and the VHI score in teachers yielded a significant inverse correlation, both with respect to the VHI-T score and with respect to the score on any subscale (functional, emotional or physical). This correlation was confirmed using both Pearson's coefficient and regression analysis (table 2). Such a correlation has not been observed in controls (euphonic females).

When the relationship between the acoustic parameters and VHI-T score was analyzed (table 3), a positive significant correlation was found in teachers for the frequency perturbation parameters (jitter, RAP, PPQ) in both statistical analyses. These findings also referred to the amplitude perturbation parameters (shimmer, APQ). Such correlations have not been observed in controls. No significant relationship between the NHR and the VHI-T score could be found in group 1, whereas an inverse correlation was observed in group 2 (table 3 ). The analysis of the relationship between the acoustic parameters and the particular VHI subscales (table 4) revealed a positive correlation with the functional subscale in teachers, in the calculations using both statistical analyses. As regards the emotional subscale, significant positive correlations were found with almost all the acoustic parameters under study (except for the NHR). For the physical subscale, no significant correlations with the acoustic parameters could be observed.

\section{Discussion}

Objective voice assessment, employing, for instance, the acoustic analysis, precludes evaluation of the biopsychosocial impact of voice disorders [15]. Therefore, the $\mathrm{VHI}$, which is used to measure the influence of voice problems on one's quality of life, offers unique information for the multidimensional diagnostics of dysphonia [16-18]. In the literature, opinions on the possible correlation between the objective and subjective parameters of voice assessment differ widely. Some authors showed a relationship between self-perceived voice problems and certain objective acoustic measures of voice [19-22]. In other studies, subjective voice complaints did not correlate with acoustic measurements [23, 24].

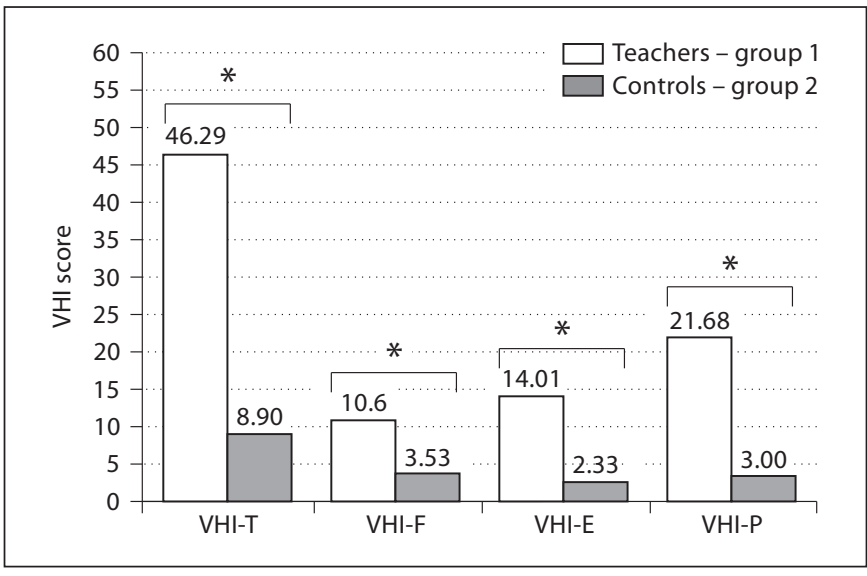

Fig. 2. The mean total score on VHI (VHI-T) and its subscales (VHI-F, VHI-E and VHI-P) in teachers and controls. ${ }^{*} \mathrm{p}<$ 0.001 .

Table 1. Acoustic parameters (mean \pm SD) in the teachers (group 1) and controls (group 2)

\begin{tabular}{lccr}
\hline Parameters & Group 1 & Group 2 & \multicolumn{1}{c}{$\mathrm{p}$} \\
\hline MPT, s & $12.2 \pm 4.53$ & $17.2 \pm 2.96$ & $<0.001$ \\
Fo mean, Hz & $217 \pm 44.64$ & $221 \pm 24.74$ & 0.090 \\
Jitter, \% & $0.33 \pm 0.09$ & $0.20 \pm 0.08$ & 0.007 \\
RAP, \% & $0.24 \pm 0.07$ & $0.12 \pm 0.05$ & 0.041 \\
PPQ, \% & $0.29 \pm 0.09$ & $0.14 \pm 0.06$ & 0.044 \\
Shimmer, \% & $4.63 \pm 1.21$ & $2.72 \pm 0.78$ & $<0.001$ \\
APQ, \% & $3.54 \pm 0.89$ & $2.07 \pm 0.56$ & $<0.001$ \\
NHR, \% & $3.49 \pm 0.42$ & $2.52 \pm 0.24$ & 0.144 \\
\hline
\end{tabular}

Fo mean $=$ Mean fundamental frequency.

Although the VHI is not the most suitable instrument for subjective assessment of professional voice, there is no better tool at this moment. It is commonly used in EU countries, including Poland, so it can be a helpful tool for the evaluation of occupational dysphonia. The present study investigated the relationship in teachers between the VHI (the total score and its particular subscales) and the objective measurements (the acoustic parameters of sustained vowel phonation and the aerodynamic parameter MPT). The study group covered female teachers, predominantly with functional dysphonia. The other subjects in this group were diagnosed with BVFM (vocal nodules and small polyps of vocal fold margin), which are assumed to be work-related vocal fold lesions due to vocal effort [25]. 
Table 2. Correlation between MPT and VHI-T, VHI-F, VHI-E and VHI-P

\begin{tabular}{lclllll}
\hline & Group 1 & & Group 2 & \\
\cline { 2 - 3 } \cline { 5 - 6 } \cline { 5 - 6 } $\begin{array}{l}\text { Pearson } \\
\text { coefficient }\end{array}$ & $\begin{array}{l}\text { regression } \\
\text { analysis }\end{array}$ & & $\begin{array}{l}\text { Pearson } \\
\text { coefficient }\end{array}$ & $\begin{array}{l}\text { regression } \\
\text { analysis }\end{array}$ \\
\hline MPT and VHI-T & $-0.273^{* *}$ & $0.003 \downarrow$ & & 0.019 & 0.922 \\
MPT and VHI-F & $-0.234^{*}$ & $0.010 \downarrow$ & & 0.096 & 0.614 \\
MPT and VHI-E & $-0.262^{* *}$ & $0.040 \downarrow$ & & -0.011 & 0.954 \\
MPT and VHI-P & $0.218^{*}$ & $0.017 \downarrow$ & & -0.064 & 0.738 \\
\hline
\end{tabular}

${ }^{*} \mathrm{p}<0.05 ;{ }^{* *} \mathrm{p}<0.01 . \downarrow=$ Negative correlation at $\mathrm{p}<0.05$.
Table 3. Correlation between acoustic parameters and VHI-T score

\begin{tabular}{llllll}
\hline \multirow{2}{*}{$\begin{array}{l}\text { Acoustic } \\
\text { parameters }\end{array}$} & Group 1 & & & \multicolumn{2}{l}{ Group 2 } \\
\cline { 2 - 3 } \cline { 5 - 6 } & P coefficient & R analysis & & P coefficient & R analysis \\
\hline Jitter & $0.199^{*}$ & $0.030 \uparrow$ & & 0.088 & 0.644 \\
RAP & $0.186^{*}$ & $0.041 \uparrow$ & & 0.100 & 0.598 \\
PPQ & $0.191^{*}$ & $0.036 \uparrow$ & & -0.116 & 0.542 \\
Shimmer & $0.262^{* *}$ & $0.004 \uparrow$ & & -0.184 & 0.330 \\
APQ & $0.243^{* *}$ & $0.007 \uparrow$ & & -0.249 & 0.184 \\
NHR & 0.147 & 0.108 & & $-0.421^{*}$ & $0.021 \downarrow$ \\
\hline
\end{tabular}

${ }^{*} \mathrm{p}<0.05 ;{ }^{* *} \mathrm{p}<0.01 . \downarrow=$ Negative correlation at $\mathrm{p}<0.05$ $\uparrow=$ positive correlation at $\mathrm{p}<0.05$.

Table 4. Correlation between acoustic parameters and VHI subscales VHI-F, VHI-E and VHI-P

\begin{tabular}{|c|c|c|c|c|c|c|c|c|c|c|c|c|}
\hline \multirow{3}{*}{$\begin{array}{l}\text { Acoustic } \\
\text { parameters }\end{array}$} & \multicolumn{6}{|l|}{ Group 1} & \multicolumn{6}{|l|}{ Group 2} \\
\hline & \multicolumn{2}{|l|}{ VHI-F } & \multicolumn{2}{|l|}{ VHI-E } & \multicolumn{2}{|c|}{ VHI-P } & \multicolumn{2}{|l|}{ VHI-F } & \multicolumn{2}{|l|}{ VHI-E } & \multicolumn{2}{|l|}{ VHI-P } \\
\hline & $\mathrm{P}$ & $\mathrm{R}$ & $\mathrm{P}$ & $\mathrm{R}$ & $\mathrm{P}$ & $\mathrm{R}$ & $\mathrm{P}$ & $\mathrm{R}$ & $\mathrm{P}$ & $\mathrm{R}$ & $\mathrm{P}$ & $\mathrm{R}$ \\
\hline Jitter & $0.229^{*}$ & $0.012 \uparrow$ & $0.190^{*}$ & $0.038 \uparrow$ & 0.095 & 0.303 & -0.152 & 0.422 & 0.355 & 0.054 & 0.101 & 0.594 \\
\hline RAP & $0.224^{*}$ & $0.014 \uparrow$ & $0.180^{*}$ & $0.049 \uparrow$ & 0.076 & 0.408 & -0.199 & 0.292 & $0.476^{* *}$ & 0.008 & 0.083 & 0.661 \\
\hline PPQ & $0.227^{*}$ & $0.012 \uparrow$ & $0.183^{*}$ & $0.045 \uparrow$ & 0.083 & 0.370 & -0.285 & 0.127 & 0.237 & 0.207 & -0.118 & 0.533 \\
\hline Shimmer & $0.294^{* *}$ & $0.001 \uparrow$ & $0.232^{*}$ & $0.011 \uparrow$ & 0.159 & 0.082 & -0.288 & 0.123 & -0.054 & 0.778 & -0.066 & 0.730 \\
\hline APQ & $0.273^{* *}$ & $0.003 \uparrow$ & $0.223^{*}$ & $0.014 \uparrow$ & 0.137 & 0.134 & -0.337 & 0.069 & -0.109 & 0.566 & -0.119 & 0.532 \\
\hline NHR & $0.197^{* *}$ & 0.031 & 0.145 & 0.114 & 0.035 & 0.707 & $-0.386^{*}$ & $0.035 \downarrow$ & -0.219 & 0.245 & -0.356 & 0.053 \\
\hline
\end{tabular}

${ }^{*} \mathrm{p}<0.05 ;{ }^{* *} \mathrm{p}<0.01 . \mathrm{P}=$ Pearson coefficient; $\mathrm{R}=$ regression analysis; $\downarrow$ = negative correlation at $\mathrm{p}<0.05 ; \uparrow=$ positive correlation at $\mathrm{p}<0.05$.

The results of this study revealed significant positive correlations between the VHI-T score, or its functional and emotional subscales, and the acoustic perturbation parameters (both frequency perturbation parameters and amplitude perturbation parameters) in the teachers. The correlations found in the teachers were confirmed by calculations using both statistical methods, namely, Pearson's correlation coefficient and regression analysis. No relationship was observed between the score on the physical subscale of the VHI and the acoustic perturbation parameters.

Similar findings were reported by Woisard et al. [21] (the acoustic parameters did not correlate with the score on the physical subscale); however, the authors observed a positive correlation with the emotional subscale and sometimes also with the functional subscale. The strong correlation between the acoustic parameters and the
VHI-F in the present study could be explained by the fact that the study group was a largely homogeneous population of female teachers (compared to the highly diverse population in the former study). The VHI is a subjective score. In a heterogeneous group, the impact of voice pathologies may be different in different persons. Probably, in a group of teachers, the impact may be similar. This observation emphasizes the dynamic interaction between voice function on the one hand and activity limitation and participation restriction on the other hand, particularly in the teaching profession.

Fulljames and Harris [26], investigating the correlation between subjective (VHI and self-rating scale of voice impairment) and objective voice outcome measures, found significant associations with the acoustic perturbation parameters in the pre-therapy measurements. They observed (just as we did) that only the sus- 
tained vowel perturbation showed a strong correlation with the VHI score. They stressed that an increase in frequency perturbation was associated with reduced voice stamina. In the post-therapy outcomes, they found a weak correlation between 'voice improvement' measurements. In our study, no significant relationship between subjective and objective voice assessment could be observed in the control group with euphonic voice. This may have been due to the fact that the low VHI-T score indicating a lack of self-perceived voice handicap may prevent a correlation with the nearly normal objective voice measures. Moreover, the comparison of relationships between the VHI score and the acoustic perturbation parameters in teachers and controls confirmed that correlations observed in teachers are not incidental.

Our study revealed no significant correlation between the NHR and the subjective measures of voice assessment (the total score of the VHI and its subscales). In the clinical picture of occupational dysphonia, the voice fatigue and reduced stamina after vocal effort (measured among other things by acoustic perturbation parameters) are prominent problems, rather than turbulent noise level at the glottis (hoarseness) measured by the NHR $[27,28]$. This may be the reason why in our study the NHR did not correlate with the VHI score.

The analysis of the relations between another objective parameter (MPT) and the VHI in the teachers yield- ed a significant inverse correlation with the total score on VHI or with any of its subscales, when both statistical methods were applied. The results of other studies [29, 30 ] indicated that the reduced respiratory capacity and decreased MPT had a negative impact on voice endurance as well as on the VHI score, which has been confirmed by the present findings.

\section{Conclusions}

In conclusion, this study revealed a significant relationship between the objective voice parameters and the VHI among the dysphonic female teachers. The results of our study confirmed that the VHI can be a valuable tool for assessing the impact of occupational dysphonia on biopsychosocial aspects of life. In reference to the WHO multidimensional concept of health as physical, mental and social well-being, the VHI should be included in the assessment of voice disorders in teachers as a valuable instrument of comprehensive voice evaluation.

\section{Acknowledgements}

This study was supported by the project of the Polish State Committee for Scientific Research, Project No 18.6/07. We thank Prof. F. de Jong for his valuable comments on this article.

\section{References}

1 Yu P, Ouaknine M, Revis J, Giovanni A: Objective voice analysis for dysphonic patients: a multiparametric protocol including acoustic and aerodynamic measurements. J Voice 2001; 15:529-542.

$\checkmark 2$ Awan SN, Roy N: Acoustic prediction of voice type in women with functional dysphonia. J Voice 2005;19:268-282.

-3 Jónsdottir V, Laukkanen AM, Vilkman E: Changes in teachers' speech during a working day, with and without electric sound amplification. Folia Phoniatr Logop 2002;54: 282-287.

4 Rubin JS, Sataloff RT, Korovin GS: Diagnosis and Treatment of Voice Disorders, ed 2. New York, Thomson Delmar Learning, 2003.

5 Steen IN, MacKenzie K, Carding PN, Webb A, Deary IJ, Wilson JA: Optimising outcome assessment of voice intervention. 2 . Sensitivity to change of self-reported and observerrated measures. J Laryngol Otol 2008;122: 46-51.
6 World Health Organization: International Classification of Impairments, Disabilities and Handicaps: A Manual of Classification, Relating to the Consequences of Disease. Geneva, World Health Organization, 1980, pp 25-43.

$>7$ Verdolini K, Ramig LO: Occupational risks for voice problems. Logoped Phoniatr Vocol 2001;26:37-46.

$>8$ Behlau M, Hogikyan D-N, Gasparini G: Quality of life and voice: study of Brazilian population using the voice-related quality of life measure. Folia Phoniatr Logop 2007;59: 286-296.

9 Sliwinska-Kowalska M, Niebudek-Bogusz E, Fiszer M, Los-Spychalska T, Kotylo P, Sznurowska-Przygocka B, Modrzewska M: The prevalence and risk factors for occupational voice disorders in teachers. Folia Phoniatr Logop 2006;58:85-101.

10 de Jonge FI, Kooijman PG, Thomas G, Huinck WJ, Graamans K, Schutte HK: Epidemiology of voice problems in Dutch teachers. Folia Phoniatr Logop 2006;58:186-198.
-11 Bovo R, Galceran M, Petruccelli J, Hatzopoulos S: Vocal problems among teachers: evaluation of a preventive voice program. J Voice 2007;21:705-722.

12 Jacobson BH, Johnson A, Grywalski C, Silbergleit A, Jacobson G, Benninger MS, Newman CW: The Voice Handicap Index (VHI): development and validation. Am J Speech Lang Pathol 1997;6:66-70.

13 Verdonck-de Leeuw I, Kuik DJ, De Bodt MS, Guimaraes I, Holmberg EB, Nawka T: Validation of the Voice Handicap Index (VHI) by assessing equivalence of European translations. 7th Pan-Eur Voice Conf, Groningen, 2007.

14 Niebudek-Bogusz E, Kotylo P, SliwinskaKowalska M: Evaluation of voice acoustic parameters related to the vocal-loading test in professionally active teachers with dysphonia. Int J Occup Med Environ Health 2007;20:25-30.

15 Maertens K, de Jong F-I: The voice handicap index as a tool for assessment of the biopsychosocial impact of voice problems. B-ENT 2007;3:61-66. 
16 Kooijman P, Thomas G, Graamans K, de Jong F: Psychosocial impact of the teacher's voice throughout the career. J Voice 2007;21: 316-324.

- 17 Rosen CA, Murry T, Zinn A, Zullo T, Sonbolian M: Voice Handicap Index change following treatment of voice disorders. J Voice 2000;14:619-623.

- 18 Speyer R, Wieneke GH, Deyonckere PH: Self-assessment of voice therapy for chronic dysphonia. Clin Otolaryngol 2004;29:6674.

19 Rantala L, Vilkman E, Bloigu R: Voice changes during work: subjective complaints and objective measurements for female primary and secondary schoolteachers. J Voice 2002;16:344-355.

20 Hsiung MW, Pai L, Wang HW: Correlation between voice handicap index and laboratory measurements in dysphonic patients. Eur Arch Otorhinolaryngol 2002;259:9799.
21 Woisard V, Bodin S, Yardeni E, Puech M: The Voice Handicap Index: correlation between subjective patient response and quantitative assessment of voice. J Voice 2007;21: 623-631.

22 Koojman PG, Wild CM, de Jong FI: The relation between the type of voice range profile and voice handicap in female voice patients. 25th Congr Union Eur Phoniatr, Poznan, 2008.

23 Wheeler KM, Colins SP, Sapienza CM: The relationship between VHI scores and specific acoustic measures of mildly disordered voice production. J Voice 2006;20:308-317.

24 Laukkanen AM, Jarvinen K, Artkowski M, Waaramaa T, Kankare E, Sipola S, Syrjä T, Salo A: Changes in voice and subjective sensations during a 45 -min vocal loading test in female subjects with vocal training. Folia Phoniatr Logop 2004,56:335-346.

25 Dejonckere PH: Occupational Voice-Care and Cure. The Hague, Kugler Publications, 2001.
6 Fulljames N, Harris S: Voice outcome measures: correlations with patients' assessment of their condition and the effectiveness of voice therapy. Logoped Phoniatr Vocol 2006; 31:23-35.

27 Zaoming Huang D, Minifie DF, Kasuya H, Xiao Lin S: Measures of vocal function during changes in vocal effort level. J Voice 1995; 4:429-438.

28 Murphy JP, McGuigan GK, Colreavy M: Investigation of a glottal-related harmonicsto-noise ratio and spectral tilt as indicators of glottal noise in synthesized and human voice signals. J Acoust Soc Am 2008;123: 1642-1652.

29 Schindler A, Bottero A, Capaccio P, Ginocchio D, Adorni F, Ottaviani F: Vocal improvement after voice therapy in unilateral vocal fold paralysis. J Voice 2008;22:113118

30 Niebudek-Bogusz E, Kotylo P, Politanski P, Sliwinska-Kowalska M: The effectiveness of voice therapy for teachers with dysphonia. Folia Phoniatr Logop 2008;60:134-141. 